\title{
SURFACE AND VOLUME HYSTERESIS OF SPUTTERED COCY
}

K. Hermes, J.C. Lodder, Th.J.A. Popma

Twente University of Technology, P.o.Box 217, 7500 AE Enschede, The Netherlands.

Abstract. Surface magnetic hysteresis, measured by polar M.o. Kerr effect on $\mathrm{RF}$ and Magnetron sputtered $\operatorname{CoCr}(81 / 19$ ato $)$ films in the range $20-4600 \mathrm{~mm}$ is compared with volume hysteresis measured by VSM, both with external field perpendicular to the surface. The surface coercivity $\mathrm{H}_{\mathrm{cs}}$ was found to decrease below the volume ooercivity at a critical thickness of $125 \mathrm{~mm}$ for RF films [I] and at about 1100 and $800 \mathrm{~mm}$ for centre and off-centre Magnetron samples respectively. The slope of the Kerr loop at $\theta_{k}=\emptyset$ increased slightly again deviating from the vSM slope at a film thickness of about 100, 800 and $500 \mathrm{~nm}$ for RF, centre and offcentre Magnetron $\operatorname{CoCr}$ samples respectively. These results are consistent with the formation of small spikes from the surface for films thicker than a critical value. Order of magnitude calculations do not oppose the formation of such small reversed domains and the higher critical thickness for Magnetron samples is also in qualitative agreement with these calculations, due to a higher perpendicular anisotropy by a factor 2 for these films. If spike domains do exist in $\operatorname{CoC} x$ the reversal mechanism is most likely one in which the reversed domains grow at the expense of the main damains.

\section{INIRODUCTION}

The reversal mechanism and associated with it the domain structure of $\mathrm{Cocr}$ thin films for perpendicular magnetic recording is not clear at present. There are two main models, namely the particulate and the continuous model. In the first ane the $\mathrm{CoCr}$ columns that are formed during film deposition are believed to interact only through magnetostatic interaction. No exchange force acts over the oolum boundaries due to e.g. a higher concentration of $\mathrm{Cr}$ there. In the continuous model the reversal mechanism is thought to take place by Bloch walls as in stripe damins, hindered by the colum boundaries [2].

of the base of Neutron Depolarisation and Magneto-Optic Kerr measurements a third intermediate model has been proposed, in which the magnetization reversal takes place by spike damain wall motion within the columns $[1,4]$.

Using the Magneto-Optic Kerr Effect (MOKE) the hysteresis loop of the surface of $\mathrm{cocr}$ can be measured, since the penetration depth of light $(\lambda=632 \mathrm{~m})$ into $\operatorname{Cor}$ is about $15 \mathrm{rm}$. comparison of surface and volume hysteresis could reveal a possible inhomogeneity in the magnetization distribution thraughout the thickness of the film and thus more insight can be gained in the domain structure and reversal mechanism of the cocr films.

Earlier we reported on the surface coercivity $\mathrm{H}_{\mathrm{Cs}}$ and the coercivity of the total film $\mathrm{H}_{\mathrm{CV}}$ measured with a VSM as a function of film thickness [1]. A rotating analyser apparatus was used to measure the Kerr rotation as a function of the applied field. The experimental set up is described in [1]. Using a maltiple-reflection sample holder the kerr rotation could be measured with an accuracy of $6.062 \mathrm{deg}$. A maximum $\mathrm{F}_{\mathrm{C}}$ was found at $t=80 \mathrm{~mm}$ for $R F-f i l m s$ and at about $100 \emptyset \mathrm{mm}$ for Magnetron films (both $81 / 19$ at 8 ). The coercivity ratio $C R$ is defined by $\mathrm{H}_{\mathrm{CS}} / \mathrm{H}_{\mathrm{CV}}$. For $\mathrm{RF}$-films a critical film thickness $t_{c}=125 \mathrm{~mm}$ was found, so that if $t<t_{c}$ then $C R>I$ and if $t>t_{C}$ then $C R<1$.

The presence of a critical thickness in Magnetron films was not clear from the reported data, therefore additional samples were Magnetron sputtered, also to cover the thicker films regions, and results are reported here. Secondly we will compare the slope of the easy-axis hysteresis loop measured with the Kerr effect and VSM at $\theta_{\mathrm{k}}=\emptyset$ and $M=\emptyset$ respectively.

The functional dependerce of the VSW slope on film thickness for RF-films was explained by Wielinga using a continuous domain model [2]. Hubert expanded the theoretical basis of this analysis to branched damain structures in $\operatorname{cocr}$ [3].

\section{SAMPLE PREPARATION AND CHARACTERIZATION}

The Cocr samples were sputtered from alloyed $\operatorname{cocr}(81 / 19$ ato $)$ targets of $4^{\prime \prime}$ and $3^{\prime \prime}$ inch dianeter respectively, on $\mathrm{Si}$ (1ø6) substrates in a Leybold Heraeus RF-sputter apparatus (ZADØ) either with or without Magnetron facilities. We will distinguish here between $\mathrm{RF}-$ and Magnetron films. RF-films were sputtered in batches of 4 samples under the optimized sputter parameters $P_{A Y}=4 \times 10^{-3}$ mRar and $V_{R F}=-1.6 \mathrm{kV}$ [5] While for Magnetron films the optimum paraneters for obtaining good perpendicular anisotropy were determined at $P_{A r}=6 \times 10^{-3} \mathrm{mBar}$ and $\mathrm{V}_{\mathrm{RF}}=-250 \mathrm{~V}$. The target substrate distance was 5 and $3 \mathrm{~cm}$ respectively. As is well know from literature sputter rate and film properties in Magnetron sputtering depend on the position of the sample on the substrate. Two hamogeneity groups were formed based on the film thickness and coercivity that coincided with a substrate position near the centre and off-centre. Here they will be referred to as group 1 and 2. The previously reported [1] Magnetron samples all belong to group 1 (near the centre). In addition to these, 5 series of 23 samples were Magnetron sputtered to cover a thickness range of 500 to $4200 \mathrm{~mm}$. Of each series 8 samples fell in group $I$ and 15 samples in group 2 . Xray fluorescence was used to cheok the composition and film thickness. The saturation magnetization $\mathrm{M}_{\mathrm{s}}$ could be determined at $460 \mathrm{kA} / \mathrm{m}$ from these measurements together with VSM results, and was found to be almost independent of film thickness. Also a constant Kerr rotation of about $\emptyset .1$ degree was found for all films, indicating that $M_{s}$ and composition do not significantly depend on film thickness. Here the (magnetic) film thickness is determined from the magnetic wolume of the sample $\left(M_{S} \cdot V\right)$ determined by VSM $( \pm 3 \%)$, the sample surface $\left(1 \mathrm{~cm}^{2}\right)$ and $M_{s}=465 \mathrm{kA} / \mathrm{m}$. The perpendicular anisotropy constant $\mathrm{K}_{1}$ corrected for the sheet
demagnetization $1 / 2 \mu \mathrm{H}_{\mathrm{s}}{ }^{2}$ was detemined from torque measurements at $90 \mathrm{~kJ} / \mathrm{m}^{3^{-}}$for RF-films [6] and now found to be $196 \mathrm{~kJ} / \mathrm{m}^{3}$ for magnetron films. No dependence on film thickness was found nor did $k_{1}$ differ significantly for Magnetron sarmles from group 1 or 2 . In preparing thick vagnetron sarples we were faced with the problem that the $\mathrm{CoCr}$ spontanuously peeled of the Si substrate due to internal stress. Presputtering the substrates at $V_{R F}=1 \mathrm{kV}$ for $10-$ 15 minutes prevented this problem. The thickest films however had to be measured quickly after preparation and still only 8 samples could be measured with both MOKE and VSM. Before peeling off occurred the metallic appearance of the $\mathrm{CoCr}$ disappeared probably due to the formation of microcracks. VSM hysteresis curves were measured both with applied field inplane and perpendicular to the surface. For all films $\mathrm{s} / /$ defined as $\left(\mathrm{M}_{\mathrm{r}} / \mathrm{M}_{\mathrm{S}}\right) / /$ was smaller than 0.12 and typically 0.09 for Magnetron and 0.06 for $\mathrm{R} F$-films, indicating good perpendicular anisotropy of the $\mathrm{CoCr}$ films.

\section{COERCIVITY}

The surface coercivity $\mathrm{H}_{\mathrm{CS}}$ for RE-films was found to decrease almost discontinuously at a critical film thickness of $125 \mathrm{~nm}$ after a maximum was reached of $120 \mathrm{kA} / \mathrm{m}$ at a film thickness of $89 \mathrm{~nm}$. For Magnetron films the results are blurred dise to the intrinsic spread in the samples (depending on substrate position). But also for Magnetron films it is found that $\mathrm{H}_{\mathrm{CS}}$ decreases more rapidly with increasing film thickness than the volume coercivity $\mathrm{H}_{\mathrm{Cv}}$ after a maximum is reached. In Fig. Ia $\mathrm{H}_{\mathrm{CS}}$ (Kerr) and $\mathrm{H}_{\mathrm{CV}}$ (ViM) are plotted as a function of film thickness. Symbols without error bars were previously published [1] each representing a single sample. The symbols with error bars inaicate the average values of film thickness and coercivity per group and the bars show the standard deviations of the coercivity. A maximm coercivity of $10 \emptyset \mathrm{kA} / \mathrm{m}(1250$ de) is found for group 1 samples at a film thickness of about $1090 \mathrm{~mm}$. The maximum coercivity $\mathrm{H} c \mathrm{c}$ for group 2 is about $90 \mathrm{kA} / \mathrm{m}(1100$ de) at a film thickness of $760 \mathrm{~mm}$ approximately. Again the surface coercivity $\mathrm{H}_{\mathrm{Cs}}$ decreases faster than $\mathrm{H}_{\mathrm{CV}}$.

This can be seen more clearly in Fig. Ib were the coercivity ratio (CR) is plotted as a function of film thickness. Here the averages of the samples from group 1 and 2 are plotted with 
different symbols to distinguish them from the previously reported samples. A critical thickness is found at about $1190 \mathrm{~mm}$ for group 1 and for group 2 (off-centre) samples the critical thickness mast certainly. lie between 400 and $1900 \mathrm{~mm}$. but probably between 780 and $1000 \mathrm{~mm}$, after the maximum $\mathrm{H}_{\mathrm{C}}$ is reached. So also for Magnetron films a critical thickness (defined by the coercivity ratio) is found. The maximum coercivity is about the same for RF and Magnetron samples, only the thickness at which it is reached is shifted to thicker films together with the critical thickness for Magnetron films.

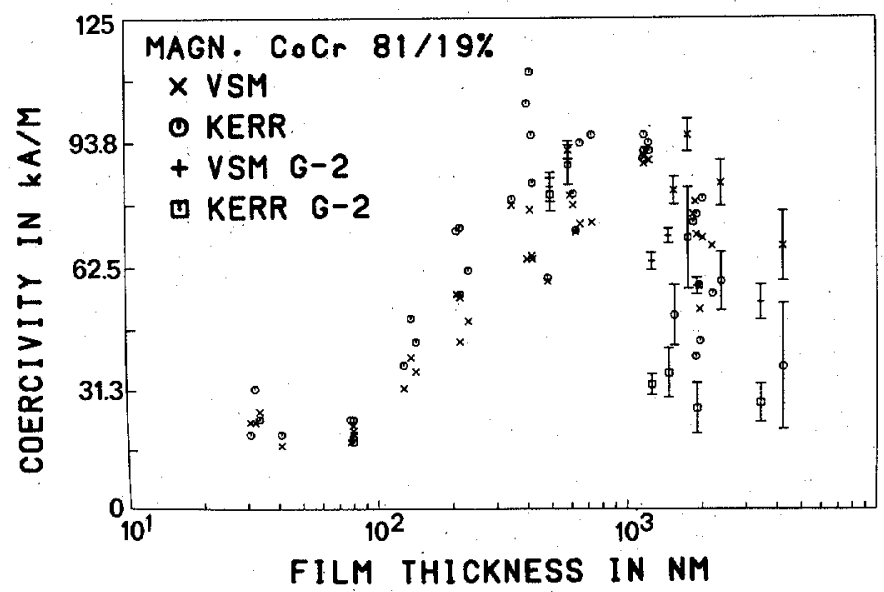

Fig. la. $\mathrm{H}_{\mathrm{CV}}$ (VSM) and $\mathrm{H}_{\mathrm{CS}}$ (Kerr) as a function of film thickness for Magnetron sputtered cocr. (see text).

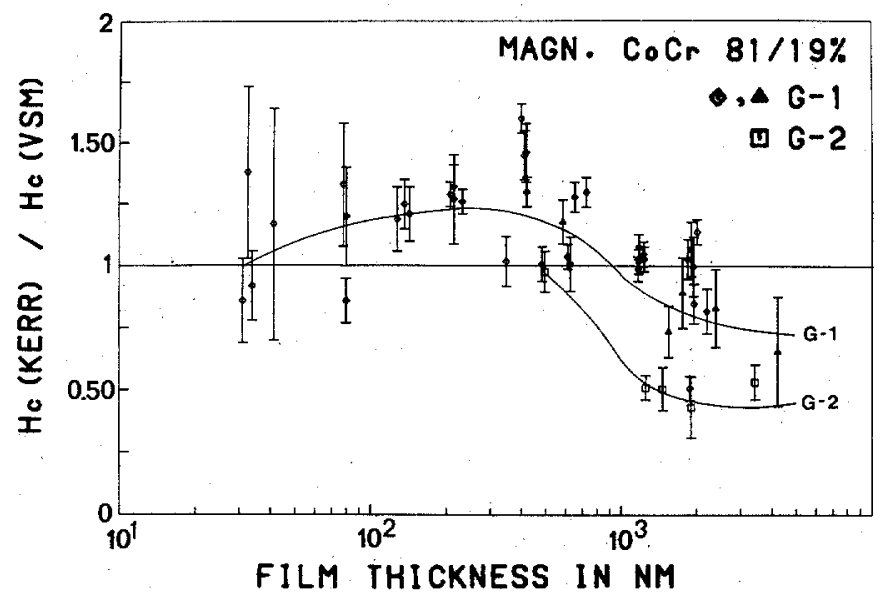

Fig. 1b. The Coercivity Ratio (C.R) versus film thickness calculated from the data in Fig. la (see text).

SLOPE OF THE PERPENDICULAR LOOP.

Rp-sputtered CoCr films.

The slope of the perpendicular hysteresis curve at. $M=\emptyset$ as a function of film thickness for RF-films was explained by Wielinga [2] using the Kooy \& Enz model. Also in the particulate model an increased slope was predicted for the thinnest films ( $t<100 \mathrm{rm}$ ) but this increase was not large enough to fit the experimental results. In the light of this discussion it is interesting to note, that the slope of the perpendicular hysteresis curve measured with MOKE deviates from the bulk value. In determinating the Kerr slope the physical state of saturation $\theta_{k}$ (sat) is set equal to $M_{s}$. In Fig. 2 both the slope of the Kerr and VSM curves are show as a function of film thickness for RF-films. The VSM slope reproduces the results by wielinga, but the Kerr-slope again increases, deviating from the VSM slope, for films thicker than about $180 \mathrm{rm}$.

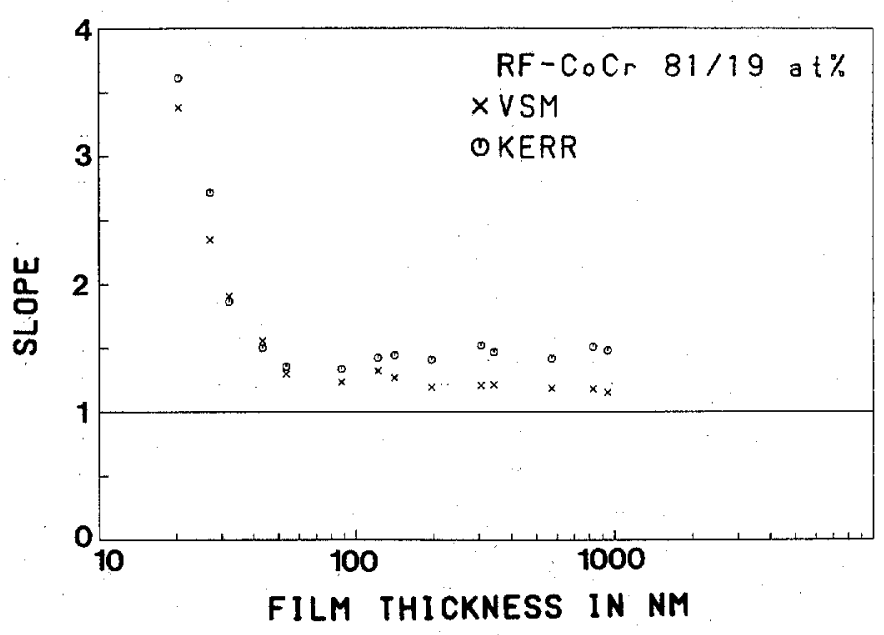

Fig. 2. The slope of the perpendicular surface (Kerr) and volume (VSM) hysteresis curve near the origin for RF-films as a function of film thickness. Fach point represents the average of 4 samples.

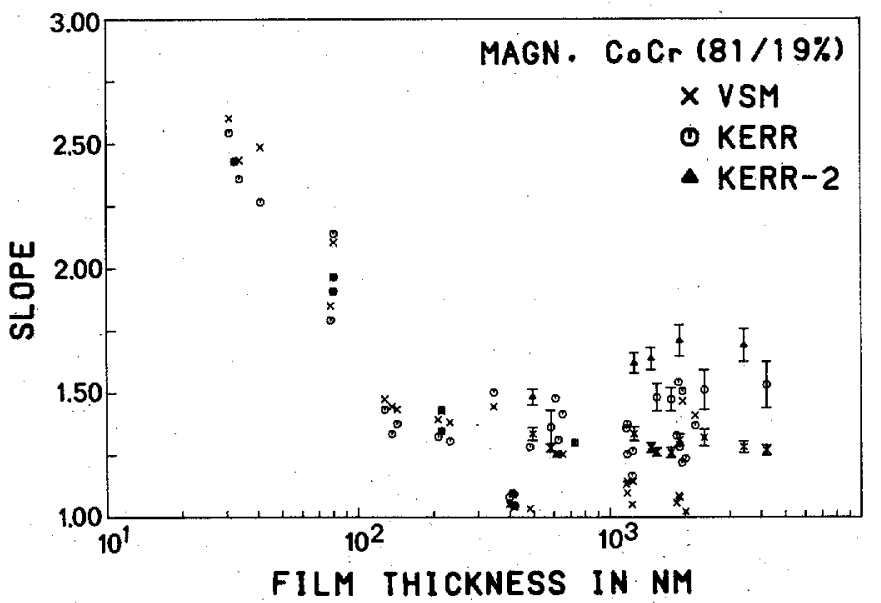

Fig. 3a. The slope of the perpendicular surface (Kerr) and volume (VSM) hysteresis curve for Magnetron films as a function of film thickness. (see text).

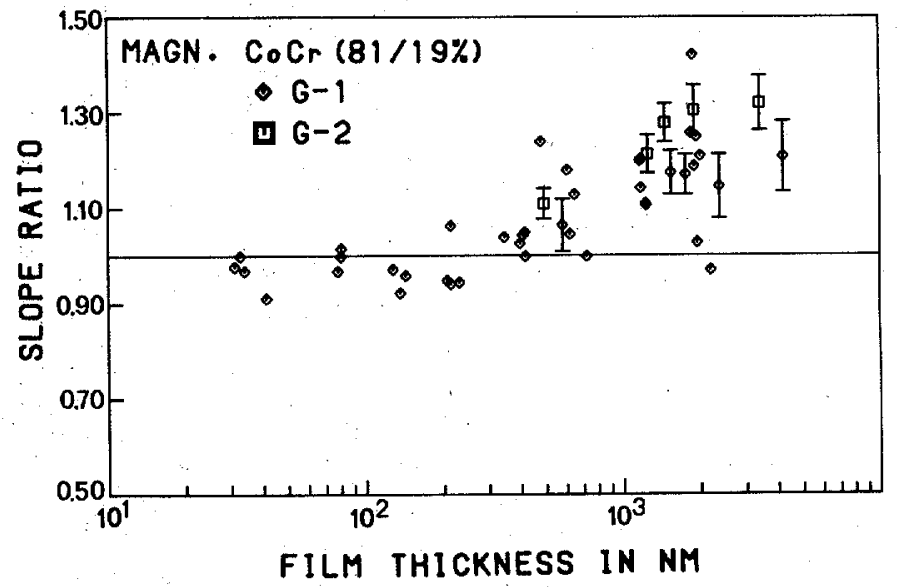

Fig. 3b. The slope ratio calculated from the data Fig. 3a versus film thickness (see text). 
Magnetron sputtered Cocr films.

Results for the Magnetron films are similar. In Fig. 3a Kerr and VSM slopes are plotted as a function of film thickness. Again the symbols without error bars refer to single samples published earlier [1] and the symbols with error bars represent the average values plus standard deviations for group 1 and group 2 samples. The VSM slope of the two groups are comparable and are both represented by a ' $x$ '. The Kerr hysteresis curve becomes significantly steeper (say 1øg) than the VSM curve for films thicker than about $8 \varnothing \varnothing$ and $5 \varnothing \varnothing \mathrm{m}$ for group I and 2 type samples respectively, as can be deduced more clearly from Fig. 3b. In this figure the ratio between the Kerr and the USM slopes calculated fram the data in Fig. $3 a$ is plotted as a function of film thickness.

\section{DISCUSSION AND CONCLUSIONS}

In Table 1 experimental values for the critical film thickness based on the coercivity ratio $t_{C}$ (C.R.) and on the Slope Ratio $t_{C}$ (S.R.) of Kerr and VSM hysteresis loops are summarized together with the film thickness $t_{\max }$ at which a maximm coercivity is reached. This $t_{\max }$ is approximately the same for $\mathrm{H}_{\mathrm{CV}}$ and $\mathrm{H}_{\mathrm{CS}}$. After the maximm is reached $\mathrm{H}_{\mathrm{CS}}$ decreases more rapidly than $\mathrm{H}_{\mathrm{CV}}$. For $\mathrm{RF}$-films this decrease is very abrupt. From $t$ is 160 to $150 \mathrm{~nm} \mathrm{H}_{\mathrm{CS}}$ decreases from 120 to $60 \mathrm{kA} / \mathrm{m}$. These results indicate that the decrease in $\mathrm{H}_{\mathrm{CV}}$ after the maximum is reached probably originates from the top surface.

At a film thickness near the maximum coercivity the slope of the Kerr hysteresis loop becomes larger than the VSM slope. These results hold as well for the RF-films as for the group I and 2 type Magnetron samples. So a relation between the three phenomena could exist.

Table 1

\begin{tabular}{|c|c|c|c|}
\hline \multirow{2}{*}{$\begin{array}{l}\text { Film thickness } \\
\text { (in } \mathrm{nm} \text { ) }\end{array}$} & \multicolumn{3}{|c|}{$\operatorname{cocr} 81 / 19$ ats } \\
\hline & $\mathrm{RF}$ & \multicolumn{2}{|c|}{ Magn. } \\
\hline & & $\mathrm{G}-1$ & $\mathrm{G}-2$ \\
\hline$t_{c}$ (C.R.) & 125 & $11 \varnothing \varnothing$ & $8 \varnothing \varnothing$ \\
\hline$t_{c}$ (S.R.) & 106 & 800 & 506 \\
\hline$t_{\max }\left(\mathrm{H}_{\mathrm{C}}\right)$ & 86 & 1000 & 700 \\
\hline
\end{tabular}

one conclusion is obvious, namely that for films with different surface and bulk hysteresis, the magnetization cannot be hamogeneous throughout the film thickness during all stages of the hysteresis curve. The cause could be a subdivision of the domains by small reversed domains at the surface (spikes), as proposed by Privorotskii [8], or a deviation of the magnetization from the perpendicular orientation as described in the $\mu^{*}$-effect. The theory must explain the different behaviour of films near the critical value. For RF-films this behaviour is almost discontinuously. Spike-domain theory is most promising, since it predicts a critical thickness at which these danains can be created to lower the total energy. According to Privorotskii these spikes penetrate to about $10 \%$ of the film thickness or not at all. The remanent magnetization $M_{x}$ is lowered at the surface only by the formation of spikes. Together with $\mathrm{M}_{\mathrm{r}}$ the coercivity $\mathrm{H}_{\mathrm{CS}}$ is decreased. The two are related by the slope $T$ according to: $M_{r}=T . H_{C}$. Hence a strong decrease in $\mathrm{H}_{\mathrm{CS}}$ accompanied by a more gradual decrease of $\mathrm{H}_{\mathrm{CV}}$ can be explained by the formation of spikes. Also an increase of the Kerr slope for films thicker than $t_{c}$ is consistent with spike domain theory. It is well known that basically the slope is equal to one, due to the sheet demagnetization;

$\mathrm{H}_{\mathrm{d}}=-\mathrm{M}$. The formation of spikes lowers the demagnetizing fileld, hence the slope of the hysteresis curve becomes steeper. Recently Hubert [3] renewed the interest in branched domain structures in thin films with uniaxial anisotropy, and also calculated the (VSM) slope of the perpendcular hysteresis curve for such a branched domain structure. Based on this calculations and the agreements with the experimental (VSM) results by Wielinga [2] (reproduced here) he concluded that his analysis confirmed the domain model of CoCr layers.

spike domains are cormonly fourd in uniaxial bulk materials like $C o$ and Magnetoplumbite [7], but these domains observed by bitter powder and Kerr microscopy are several orders of magnitude larger than expected in CoCr thin films. However, the parameter that determines the material length $\lambda_{\mathrm{m}}=\sigma_{\mathrm{w}} / \mu_{\sigma_{\mathrm{M}}}{ }^{2}\left(\sigma_{\mathrm{w}}=4\left(\mathrm{~A} \cdot \mathrm{K}_{1}\right)^{1 / 2}\right)$

is indeed very small for $\operatorname{Cocr}(81 / 19$ at 8$)$, namely $3.5 \mathrm{~nm}$ for RF-films and $5 \mathrm{~mm}$ for Magnetron films. These values have been calculated assuming that the exchange ocnstant $A$ determined for FF films at $A=6 \times 10^{-13} \mathrm{~J} / \mathrm{m}^{3}$ [2] also holds for Magnetron films. However, the value of $\lambda_{m}$ as calculated above is comparable with the dimensions of the discontinuities in the morphology of CoCr.

Using Privorotskii's theory the critical film thickness for the formation of spikes is calculated between 25 and $4 \varnothing \mathrm{nm}_{r}$ but strictly speaking this cannot be correct since than the length of the spikes would become smaller than the wall thickness. Probably the weak anisotropy assumption and the approximation of no interaction between top and bottam surface made by Privorotskii does not hold for $\mathrm{CoCr}$ in this case.

In the particulate model the critical radius $r_{C}$ for a spherical single domain particle is also very small. According to Chicazumi [7] $r_{C}=4.5 \lambda_{m}$. The column diameter $d$ and film thickness $t$ for ( $\mathrm{RF}$ ) $\mathrm{COCr}$ filins are roughly related by $d=t^{2 / 3}$ (both in $\mathrm{nm}$ ) $[6,9]$. Here we measured by (S)TEM that about the same relation holds for Magnetron films. So by sinply putting $2 r_{c}=d$ the critical film thickness would be $t_{c}=\left(9 \lambda_{m}\right)^{3 / 2}$ or $180 \mathrm{~nm}$ for $R F$ and $306 \mathrm{rm}$ for Magnetron films. Hence order of magnitude calculations do not oppose the formation of such small spike domains in $\mathrm{COCr}$ thin films. Also the larger critical film thicknesses found for Magnetron films are qualitatively predicted.

Due to the discontinuous character of the reported results, especially the surface coercivity of RF-films, it is not likely that the $\mu^{\star}$-effect will be able to explain all the results. However, a possible contribution of this effect cannot be disregarded.

Concluding, it can be stated that present results of comparing surface and bulk magnetic behaviour are consistent with a spike domain theory in Cocr thin films. If spike domains do exist in Cocr, then the reversal mechanism is most likely one in which the reversed domains grow at the expense of the main domain.

\section{ACKNOWLEDGEMENIS}

The authors would like to thark J.D. Baxter and J. Yntema for their contribution to this work.

\section{REFERENCES}

1) K. Hemmes, W. Lisowski, J.C. Lodder, L.J. Hanekamp, Th. J.A. Popma, J. Phys. D:Appl. Phys. 19, pl311-1326, 1986.

2) T. Wielinga, J.C. Lodder and J. Worst.

IEEE Trans. on Magn. Vol. MAG-18, No 6, pllø7, 1982.

3) A. Hubert.

IEEE Trans. on Mágn. Voi. MAG-21, No 5, p1694, 1985.

4) K. Hermes, J.C. Lodder, M.Th. Rekvelat, W.H. Kraan. Proceedings of the IOMFS 1985, Ansilomar U.S.A.

5) J.C. Lodder, T. Wielinga. IEEE Trans. on Magn. Vol. MAG-20, No. 1, January 1984.

6) J.C. Lodder, T. Wielinga, J. Worst. Thin Solid Films 1Ø1, p61-81, 1983.

7) S. Chicazumi "Physics of Magnetism", John Wiley \& Sons,1964. Chapter 11 .

8) I. Privosotskii, "Thermodynamic Theory of Damain Structures", John Wiley \& Sons 1976.

9) K.Hermes, J.C.Lodder, M.Th. Rekveldt, W.H. Kraan I. of Phys. D: Appl. Phys., 17, pL157-162, 1984. 